\title{
聚甲基丙烯酸酯人工晶状体表面水蛭素修饰 及抗黏附
}

\author{
郑志雯 ${ }^{(1)}$ ，焦燕 ${ }^{(1)}$ ，任力 ${ }^{(1)}$ ，王迎军 (1) (2) \\ (1) 华南理工大学材料科学与工程学院, 广州 510641; \\ (2) 国家人体组织功能重建工程技术研究中心, 广州 510006 \\ *E-mail: zzw01wind@163.com; imwangyj@163.com
}

收稿日期: 2013-11-07; 接受日期: 2014-01-10

国家重点基础研究发展计划(“973”计划)(批准号: 2012CB619104)、广东省重大科技专项(批准号: 2012A080203011)和广东省自然科学基金 (批准号: S2012010010411)资助项目

\begin{tabular}{|c|c|}
\hline 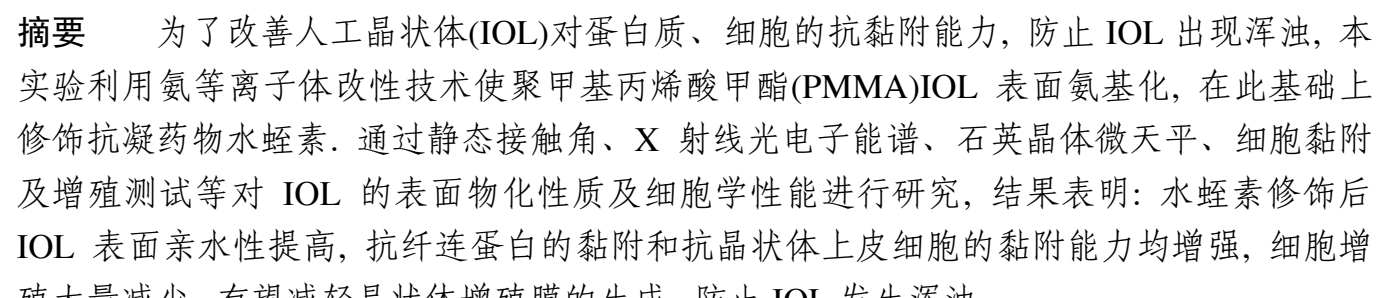 & $\begin{array}{l}\text { 关键词 } \\
\text { 聚甲基丙烯酸酯 } \\
\text { 人工晶状体 } \\
\text { 氨等离子体 } \\
\text { 水蛭素 } \\
\text { 抗稆附 }\end{array}$ \\
\hline
\end{tabular}

\section{1 引言}

聚甲基丙烯酸甲酯类人工晶状体 (poly(methyl methacrylate) intraocular lens, PMMA IOL)由于其优 越的透明度、强度、韧性、化学稳定性, 且价格较其 他种类 IOL 低廉 ${ }^{[1,2]}$, 现广泛应用在临床上. 但是植 入 PMMA IOL 后, 由于生物相容性问题, 一方面会 引发炎症异物反应; 另一方面, 晶状体上皮细胞 (LECs)的黏附、增殖、迁移和蛋白质的黏附变性诱发 增殖膜的生成, 会造成 IOL 浑浊 ${ }^{[3]}$.

近年来, 研究者们对 IOL 发生二次浑浊的问题 进行了大量研究, 如对表面进行等离子体处理 ${ }^{[46]}$ 或 接枝聚合物 ${ }^{[710]}$, 通过空间效应和水屏障效应来阻挡 蛋白质和细胞的黏附, 减少炎症反应; 或者在表面涂 覆或接枝一些如光敏剂、蛋白质或抗炎症的活性药物,

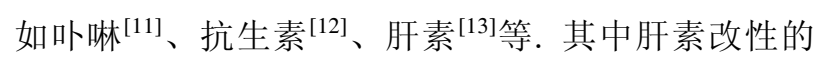
IOL 研究较为广泛, 主要利用肝素本身的消炎抗菌作 用、抗凝血作用和电荷作用来提高材料的亲水性、生 物相容性和血液相容性, 但一些研究者经过长期的 动物实验和临床效果观察表明, 肝素改性并没有有 效提高 IOL 抑制浑浊的能力 ${ }^{[14,15]}$.

等离子体处理技术常用于在材料表面引入活性 基团如氨基化来提高亲水性和生物相容性 ${ }^{[16,17]}$. 为 了进一步提高丙烯酸酯材料的亲水性, 改善材料表 面的生物功能, 常在氨基化的基础上接枝亲水性生 物大分子或功能化基团. 重组水蛭素是一种由 65 66 个氨基酸组成的小分子蛋白质(单链多肽), 是典型的 抗凝血酶药物. 有研究发现, 该抗凝蛋白除能静脉注 射抑制血栓形成外，还能通过眼部黏膜吸收用于防 治白内障 ${ }^{[18,19]}$, 因此有可能成为一种抗白内障新药.

引用格式: 郑志雯, 焦燕, 任力, 等. 聚甲基丙烯酸酯人工晶状体表面水蛭素修饰及抗黏附. 中国科学: 技术科学, 2014, 44: 603-609 Zheng Z W, Jiao Y, Ren L, et al. Anticoagulant r-hirudin coating on PMMA intraocular len surface and its nonfouling properties (in Chinese). Sci Sin Tech, 2014, 44: 603-609, doi: 10.1360/092013-1149 
同常用的 IOL 表面修饰抗凝药物肝素相比, 水蛭素 具有以下几个优点: 第一, 抑制凝血酶的反应不需要 其他凝血酶作为辅助因子, 使其抗凝作用与量效关 系更吻合; 第二, 不受血小板或糖蛋白等因素的影响; 第三, 用于抗血栓治疗, 出血副作用较小, 且无过敏 反应和免疫原性无毒反应 ${ }^{[20,21]}$.

本文在 PMMA 表面氨等离子体改性的基础上, 将小分子抗凝蛋白药物——重组水蛭素 $(\mathrm{rH})$ 结合到 材料表面. 研究利用氨等离子体处理使水蛭素药物 在材料表面形成生物涂层, 考查水蛭素修饰后材料 表面对纤连蛋白及晶状体上皮细胞的抗黏附效果. 为人工晶状体材料的产业升级和临床应用提供理论 与实验依据, 具有广阔的应用前景及重要的社会经 济价值.

\section{2 实验}

\section{1 试剂与仪器}

PMMA IOL(1 片式, 珠海艾格医疗科技开发有 限公司); PMMA 粒料(纯度 99\%); $\mathrm{rH}$ (纯度 95\%, 大连 保税区联合博泰生物技术有限公司); 人晶体上皮细 胞(HLEpiC)(ScienCell 研究实验室); 异硫氧酸荧光素 (FITC, 型号 Amresco M1425-2, 北京冬歌生物科技 有限公司); CCK-8 试剂盒(东仁化学科技(上海)有限 公司).

采用 OCA40 Micro 型静态接触角测量仪(德国 Dataphysics 公司)分析表面亲水性及各种表面能; 采 用 Kratos Axis UltraDLD 型 X 射线光电子能谱仪(XPS, 日本岛津公司)分析表面元素变化; 采用 Q-sense E4 型石英晶体微天平 (QCM-D, 瑞典凯戈纳斯有限公司) 分析表面蛋白质黏附行为; 细胞黏附形态观察采用 Nikon eclipse80i 型倒置荧光相差显微镜 (日本 Nikon 公司); 细胞增殖测定采用 HTS700 型酶标仪 (Bio Assay 公司).

\subsection{PMMA 表面氨基化及修饰水蛭素}

将 IOL 依次浸泡在 50\%酒精和去离子水中超声 清洗 $10 \mathrm{~min}$, 在鼓风干燥箱中干燥后放入等离子体 处理仪中处理, 处理条件为: 通入氨气后压力保持在 22 27 Pa, 功率在 60 80 W, 处理时间为 $5 \mathrm{~min}$, 等离 子体处理完后继续通入氨气 $10 \mathrm{~min}$, 得到氨气等离 子体处理的样品. 由于在此过程表面产生氨离子
$\left(\mathrm{NH}_{3}{ }^{+}\right)$，也称为氨基化过程. 然后将其在 $500 \mu \mathrm{g} / \mathrm{mL}$ $\mathrm{rH}$ 的 PBS 溶液中 $(\mathrm{pH} 7.4)$ 浸泡 $4 \mathrm{~h}$, 取出后用去离子 水冲洗 3 次, 真空干燥 $24 \mathrm{~h}$, 得到具有水蛭素涂层的 样品(plas-rH).

\section{3 纤连蛋白黏附行为表征}

将 PMMA 的粒料溶解在二氯甲烷中, 质量浓度 为 $3 \%$, 取 $100 \mu \mathrm{L}$ 溶液旋涂在石英晶片上, 过夜后, 晶片的表面改性处理同上.

向 QCM-D 测量样品池中顺序通入水、PBS 缓冲 液、纤连蛋白 PBS 溶液(缓冲液, $\mathrm{pH}$ 7.4)、PBS 缓冲 液、水, 每个过程均要达到平衡后才能进行下一个过 程. 利用仪器自带的 Q-Tools 分析工具根据频率和耗 散数据对表面蛋白吸附情况进行计算分析.

\section{4 细胞黏附增殖实验}

将传代培养处于对数生长区的 HLEpiC 用胰酶 消化成单个细胞悬液后, 加入含 $10 \%$ 胎牛血清的 DMEM 培养基中, 调细胞浓度至 $5 \times 10^{4}$ 个 $/ \mathrm{mL}$. 用于 细胞实验的 IOL 利用 $75 \%$ 酒精浸泡灭菌后，再进行上 述等离子体处理和 $\mathrm{rH}$ 的接枝, 整个过程在无菌环境 下进行. 样品制备完成之后置于 48 孔板中, 用 PBS 溶液冲洗 3 次并加入 $500 \mu \mathrm{L}$ 细胞悬液, 置于 $37^{\circ} \mathrm{C}$, $5 \% \mathrm{CO}_{2}$ 培养箱中培养. 分别于 $1,3,5,7 \mathrm{~d}$ 后取出, 除 去培养液, 用 PBS 缓冲液清洗 2 遍, 将样品取出放入 新的孔板中.

1) 每组取 5 个样品, 每孔加入 $200 \mu \mathrm{L}$ 提前配好 的 CCK-8 溶液(培养液: CCK- $8=10: 1$ ), 置于培养箱中 培养 2 h. 之后每孔吸取 $100 \mu \mathrm{L} \mathrm{CCK} 8$ 溶液至 96 孔板 中, 立即用酶标仪测定 $450 \mathrm{~nm}$ 处的吸光值.

2) 每组取 3 个样品, 每孔加入 $200 \mu \mathrm{L}$ 提前配好 的 FITC 苂光染色溶液, 在 $37^{\circ} \mathrm{C}$ 避光放置半小时进行 荧光标记. 标记后的样品利用 PBS 冲洗 3 次, 取出后 利用倒置荧光相差显微镜蓝光模式下观察细胞形态.

\section{3 结果与讨论}

\section{1 表面接触角及能量变化}

表面改性前后的样品对水、乙二醇(EG)、二碘甲 烷 $\left(\mathrm{CH}_{2} \mathrm{I}_{2}\right)$ 的接触角结果以及相应的极性分量、色散分 量和表面能结果如表 1 中所示. 未处理前的 PMMA 高分子链的侧链为酯基，主链由烷基链构成，极性基 
表 1 PMMA 修饰前后表面接触角及表面能

\begin{tabular}{|c|c|c|c|c|c|c|}
\hline \multirow{2}{*}{ Material } & \multicolumn{3}{|c|}{ Contact angle $\left({ }^{\circ}\right)$} & \multirow{2}{*}{$\gamma_{\mathrm{SV}}^{\mathrm{p}}\left(\mathrm{mJ} / \mathrm{m}^{2}\right)$} & \multirow{2}{*}{$\gamma_{\mathrm{SV}}^{\mathrm{d}}\left(\mathrm{mJ} / \mathrm{m}^{2}\right)$} & \multirow{2}{*}{$\gamma_{\mathrm{SV}}\left(\mathrm{mJ} / \mathrm{m}^{2}\right)$} \\
\hline & Water & EG & $\mathrm{CH}_{2} \mathrm{I}_{2}$ & & & \\
\hline PMMA & $64.15 \pm 1.277$ & $54.925 \pm 2.546$ & $66.3 \pm 5.46$ & 21.47 & 16.84 & 38.31 \\
\hline Plasma & $17.675 \pm 0.834$ & $8.075 \pm 1.461$ & $12.6 \pm 1.378$ & 37.3 & 34.53 & 71.83 \\
\hline plas-rH & $46.075 \pm 1.914$ & $21.725 \pm 2.451$ & $31.4 \pm 2.183$ & 24.9 & 32.39 & 57.29 \\
\hline
\end{tabular}

团较少, 水接触角为 $64.15^{\circ}$ 左右, 极性分量 $\gamma_{\mathrm{SV}}{ }^{\mathrm{p}}$ 较小, 亲水性较差; 经氨等离子体处理后水接触角迅速下 降为 $17.675^{\circ} \pm 0.834^{\circ}$, 极性分量 $\gamma_{\mathrm{SV}}{ }^{\mathrm{p}}$ 从 $21.47 \mathrm{~mJ} / \mathrm{m}^{2}$ 增 加到 $37.3 \mathrm{~mJ} / \mathrm{m}^{2}$, 说明氨等离子体处理可以产生大量 极性基团或离子, 这些基团和离子与水分子相互作 用使接触角迅速下降; 在形成水蛭素涂层后, 样品的 水接触角又增大至 $46.075^{\circ} \pm 1.914^{\circ}$, 并且极性分量 $\gamma_{\mathrm{SV}}{ }^{\mathrm{p}}$ 下降, 这是由于水蛭素多肽结合并覆盖在材料表 面使极性基团和离子减少, 但水蛭素分子亦含有酰 胺键和离子, 故 plas-rH 的极性分量依然比 PMMA 要 大. PMMA 的表面能 $\gamma_{\mathrm{SV}}$ 为 $38.31 \mathrm{~mJ} / \mathrm{m}^{2}$, 等离子体处 理后增加至 $71.83 \mathrm{~mJ} / \mathrm{m}^{2}$, 说明等离子体处理后材料
表面的能量不稳定，容易与外界发生反应. 结合水蛭 素后, $\gamma_{\mathrm{SV}}$ 又下降至 $57.29 \mathrm{~mJ} / \mathrm{m}^{2}$, 说明表面涂层后能 量趋于稳定, 但水蛭素分子本身含有的极性基团和 离子导致表面能不会低于 PMMA 的表面能.

\section{2 表面元素分析}

图 1 为末处理的 PMMA、等离子体处理和水蛭 素涂层修饰后的样品的 XPS 全谱图. 未处理的 PMMA 材料表面仅含微量 $\mathrm{N}$ 元素, 在氨气等离子体 处理后出现大量 $\mathrm{N}$ 元素, 说明氨气等离子体处理可 以在 PMMA 表面引入大量含 $\mathrm{N}$ 基团; 水蛭素涂层修 饰后 $\mathrm{N}$ 含量从 $8.17 \%$ 减少至 $5.7 \%$, 并且在 $166 \mathrm{~nm}$ 处
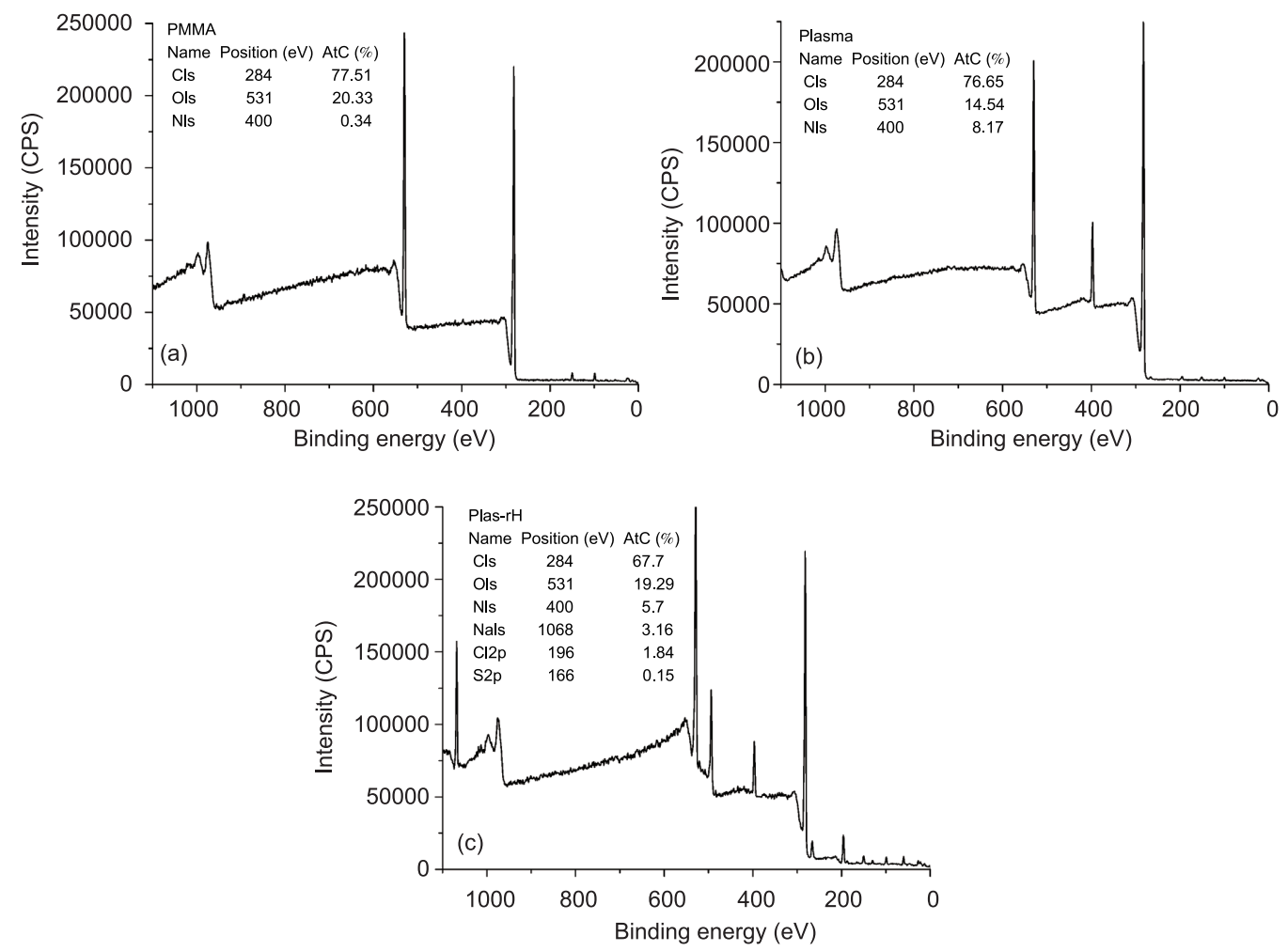

图 1 PMMA 修饰前后表面元素 XPS 全谱

(a) 处理前; (b) 氨基化后; (c) 水蛭素修饰后 
出现了新的 S 元素的吸收峰, 说明水蛭素分子成功修 饰在材料表面. plas-rH 表面在 196 和 $1068 \mathrm{~nm}$ 处有 $\mathrm{Cl}$ 和 $\mathrm{Na}$ 的峰, 这主要是由于在结合水蛭素时, 水蛭 素溶液中的 PBS 缓冲液残余的无机元素.

氨基化处理前后的 PMMA 膜表面元素周围的化 学环境也发生了改变. 将材料表面元素含量及各元 素的价态分析列入表 2, 分析 PMMA 表面修饰前后 元素化学组成及含量变化. PMMA 具有 3 个 $\mathrm{C}$ 元素的 峰, 分布在 $284.6,286.0$ 和 $288.6 \mathrm{eV}$ 左右, 分别属于 $\mathrm{C}-\mathrm{H},-\mathrm{C}-\mathrm{O}$ 和 $-\mathrm{C}=\mathrm{O}$ 的拟合峰; 氨基化后在 399.0 和 $400.0 \mathrm{eV}$ 左右产生含 $\mathrm{N}$ 元素的 $-\mathrm{C}-\mathrm{N}$ 和 $-\mathrm{NH}_{3}{ }^{+}$新峰.

对比 3 种样品的 C 元素含量及其峰归属可知, C 含量在改性后不断减小, 在 PMMA 表面的 C 元素中 $\mathrm{C}-\mathrm{H}$ 占 $67 \%, \mathrm{C}-\mathrm{O}-$ 占 $21 \%, \mathrm{C}=\mathrm{O}$ 占 $12 \%$, 这是由 PMMA 的聚合物链的结构决定的, 在 PMMA 分子链 中 $\mathrm{C}-\mathrm{O}$ 与 $\mathrm{C}=\mathrm{O}$ 的比例应为 $1: 1$, 但由于 $\mathrm{C}-\mathrm{O}$ 作为侧 链的末端覆盖在材料表面, 因此 XPS 测得的结果含 量要高一些; 在 plasma 的 C 元素中, $285.8 \mathrm{eV}$ 处的峰 含量增加到 $35 \%$, 这是由于氨等离子体处理后表面 产生大量氨基与 $\mathrm{C}-\mathrm{H}$ 反应致使 $\mathrm{C}-\mathrm{N}$ 含量增加; 而在 Plas-rH 的表面 C 元素中 C-N 含量又下降至 $21 \%$, 而

表 2 PMMA 修饰前后表面元素及其价态分析

\begin{tabular}{|c|c|c|c|}
\hline 样品 & 元素 & 价态归属 & 面积 (\%) \\
\hline \multirow{5}{*}{ PMMA } & \multirow{3}{*}{$\mathrm{C}_{1 \mathrm{~s}}$} & $-\mathrm{C}-\mathrm{H}$ & 67 \\
\hline & & $-\mathrm{C}-\mathrm{O}-$ & 21 \\
\hline & & $\mathrm{C}=\mathrm{O}$ & 12 \\
\hline & \multirow{2}{*}{$\mathrm{O}_{1 \mathrm{~s}}$} & $\mathrm{C}=\mathrm{O}$ & 51 \\
\hline & & $-\mathrm{C}-\mathrm{O}-$ & 49 \\
\hline \multirow{8}{*}{ Plasma } & \multirow{3}{*}{$\mathrm{C}_{1 \mathrm{~s}}$} & $-\mathrm{C}-\mathrm{H}$ & 58 \\
\hline & & $-\mathrm{C}-\mathrm{O}-$ 或 C-N & 35 \\
\hline & & $\mathrm{C}=\mathrm{O}$ 或 $\mathrm{N}-\mathrm{C}=\mathrm{O}$ & 7 \\
\hline & \multirow{3}{*}{$\mathrm{O}_{1 \mathrm{~s}}$} & $\mathrm{C}=\mathrm{O}$ & 33 \\
\hline & & $\mathrm{N}-\mathrm{C}=\mathrm{O}$ & 45 \\
\hline & & $-\mathrm{C}-\mathrm{O}-$ & 22 \\
\hline & \multirow{2}{*}{$\mathrm{N}_{1 \mathrm{~s}}$} & $\mathrm{~N}-\mathrm{C}=\mathrm{O}$ 或 $\mathrm{C}-\mathrm{N}$ & 37 \\
\hline & & $-\mathrm{NH}_{3}^{+}$ & 63 \\
\hline \multirow{8}{*}{ Plas-rH } & \multirow{3}{*}{$\mathrm{C}_{1 \mathrm{~s}}$} & $-\mathrm{C}-\mathrm{H}$ & 68 \\
\hline & & $-\mathrm{C}-\mathrm{O}-$ 或 C-N & 21 \\
\hline & & $\mathrm{C}=\mathrm{O}$ 或 $\mathrm{N}-\mathrm{C}=\mathrm{O}$ & 11 \\
\hline & \multirow{3}{*}{$\mathrm{O}_{1 \mathrm{~s}}$} & $\mathrm{C}=\mathrm{O}$ & 25 \\
\hline & & $\mathrm{N}-\mathrm{C}=\mathrm{O}$ & 48 \\
\hline & & $-\mathrm{C}-\mathrm{O}-$ & 27 \\
\hline & \multirow{2}{*}{$\mathrm{N}_{1 \mathrm{~s}}$} & $\mathrm{~N}-\mathrm{C}=\mathrm{O}$ 或 $\mathrm{C}-\mathrm{N}$ & 44 \\
\hline & & $-\mathrm{NH}_{3}^{+}$ & 56 \\
\hline
\end{tabular}

$\mathrm{N}-\mathrm{C}=\mathrm{O}$ 含量增加至 $11 \%$, 这可能是由于水蛭素分子 和氨基化 PMMA 表面发生作用生成酰胺基团. 同样 由于氨等离子体产生的氨基和水蛭素的接枝, $\mathrm{O}$ 元素 总含量先下降又上升; 其中 $\mathrm{C}=\mathrm{O}$ 的实际含量 $(\mathrm{C}=\mathrm{O}$ 的 含量乘以 $\mathrm{O}$ 元素总含量) 是不断增加的, 这也与 $\mathrm{N}-\mathrm{C}=\mathrm{O}$ 的生成相关. 同理由于接枝水蛭素材料表面 $\mathrm{N}$ 元素减少, 且 $\mathrm{N}-\mathrm{C}=\mathrm{O}$ 增加.

\section{3 纤连蛋白黏附行为分析}

纤连蛋白 $(\mathrm{Fn})$ 是造成二次白内障的主要蛋白, 并 且据研究者研究发现, 它也是介导细胞黏附的主要 蛋白, 为细胞的黏附提供位点. 纤连蛋白在表面黏附 的情况是预防 IOL 混浊的重要参数. 图 2 为振动频率 变化与时间 $(\Delta F-T)$ 的关系和耗散因子变化与时间 $(\Delta D-T)$ 的关系, $\Delta F$ 反映表面黏附 $\mathrm{Fn}$ 的质量变化, $\Delta D$ 反映黏附 $\mathrm{Fn}$ 的密度.

首先向测量样品池中分别通入 $\mathrm{H}_{2} \mathrm{O}$ 和 PBS 缓冲 液直至基线达到平衡, 然后通入 $\mathrm{Fn}$ 溶液. 这时 $\Delta F$ 迅 速下降, 在 $25 \mathrm{~min}$ 后下降速率放缓, 并最终在 $50 \mathrm{~min}$ 时基本达到吸附-解吸附平衡. 可以看出, 最终 $|\Delta F|$ 的 大小依次是: plasma $>$ PMMA $>$ plas-rH, 说明氨气等离 子体处理后材料对 Fn 的吸附大于未处理前的 PMMA, 而水蛭素涂层修饰的材料对 Fn 的吸附比前二者要大 大减少. 在 Fn 的吸附达到平衡后再次通入 PBS 溶液 和水冲洗可逆吸附的 $\mathrm{Fn}$, 最终 $|\Delta F|$ 的大小为: plasma $>$ PMMA $>>$ plas-rH, 说明水蛭素修饰的 PMMA 表面 Fn 的可逆吸附大量减少, 这预示着 PMMA

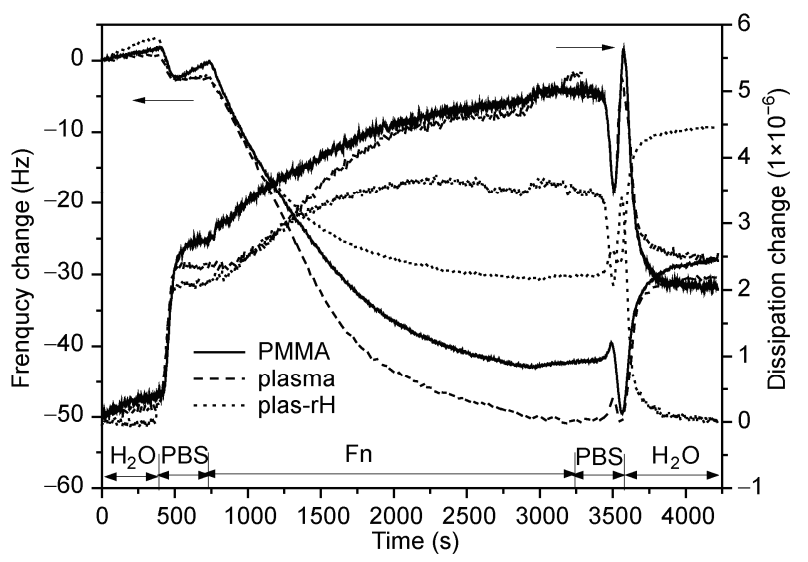

图 2 水蛭素修饰前后 PMMA 膜在第 5 倍频下 Fn 吸附过 程频率 $\boldsymbol{F}$ 和耗散 $\boldsymbol{D}$ 随时间的变化 
IOL 表面可防止 Fn 的黏附, 进而对 HLEpiC 的黏附有 一定抵抗作用.

\section{4 晶状体上皮细胞黏附分析}

IOL 表面细胞黏附形成的增生膜是影响 IOL 透 明度的主要原因, 对 HLEpiC 在材料上的黏附和增殖 情况进行体外模拟是研究抑制 IOL 二次白内障的重 要方法. 图 3 为 PMMA 和表面改性后 HLEpiC 的增 殖情况. 可以看出, 第 1 天时 3 种样品表面的细胞黏 附数量差别较小, 随时间增加细胞数量差别变大. 第 5 天时氨气等离子体处理和水蛭素涂层修饰的材料上 细胞数量大量减少, 说明亲水性可以抑制细胞的黏 附和增殖; 水蛭素涂层修饰后的细胞数量比起氨气 等离子体处理后又有下降, 结合材料对 Fn 黏附的分 析, 说明水蛭素涂层通过抑制 Fn 的黏附降低了对细 胞的介导作用, 从而抑制细胞的黏附和增殖. 第 7 天 时 PMMA 表面的细胞继续增殖, 数量不断增加, 氨

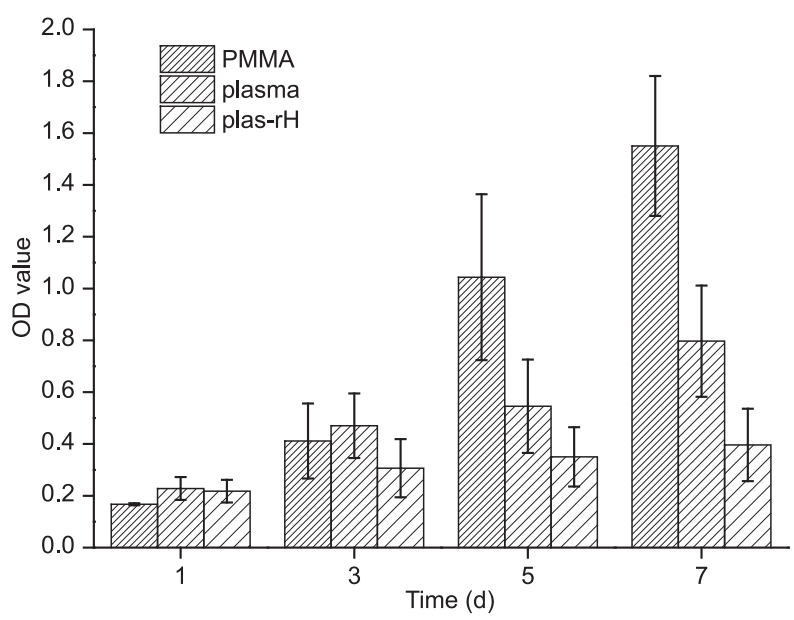

图 3 水蛭素修饰前后的 PMMA 不同时间细胞的增殖情况
气等离子体处理的材料表面细胞也略有增加, 而水 蛭素涂层修饰的材料表面细胞数量变化不大, 说明 水蛭素涂层修饰的材料对细胞的抑制作用较强.

图 4 是第 5 天表面改性前后材料上黏附细胞的荧 光染色图片. PMMA 上的细胞在表面任意铺展, 有一 些细胞伸出较长伪足攀附在材料表面, 细胞基本遍 布整个视野, 数量很多. 氨气等离子体处理的材料上 的细胞数量较少, 大多呈圆形, 有伸出的伪足但攀附 面积较小. 水蛭素涂层修饰的材料上细胞数量极少, 散落地分布在材料表面。水蛭素接枝后的 PMMA-IOL 明显比只氨基化、未处理的表面细胞黏 附少, 没有出现导致 IOL 浑浊的增生膜产生. 细胞黏 附实验结果表明，水蛭素接枝后的材料表面具有一 定的抑制细胞过度增殖能力, 比单纯氨基化处理更 能抑制细胞黏附，从而减少非特异性蛋白吸附的发 生率.

\section{4 结论}

PMMA 膜在氨等离子体激发下表面被活化，其 分子链中的酯键可能发生部分断裂生成不稳定 $\mathrm{R}-\mathrm{CO}$ 自由基，与水蛭素分子末端的 $-\mathrm{NH}_{2}$ 结合，形成 酰胺键. 因此, 本文利用酰胺反应将水蛭素分子修饰 在氨基化后的 PMMA 表面, 提高了抗非特异性蛋白 Fn 的吸附能力. 水蛭素修饰后的 PMMA 表面形成大 量 $\mathrm{N}-\mathrm{C}=\mathrm{O},-\mathrm{COO}^{-},-\mathrm{OH}$ 以及 $-\mathrm{NH}_{3}{ }^{+}$极性基团, 引起 接触角降低, 表面能减小并趋于稳定. 利用 QCM 考 察材料表面抗蛋白质吸附的动态行为. 氨基化后的 PMMA 表面吸附 Fn 的速率较快, 结合比较牢固, 为 不可逆吸附; 氨基化后的表面吸附质量较未处理前 增多, 这可能是由于氨基化后处理的表面产生的 $\mathrm{NH}_{3}{ }^{+}$离子较多, 与带负电的 $\mathrm{Fn}$ 通过静电作用结合;
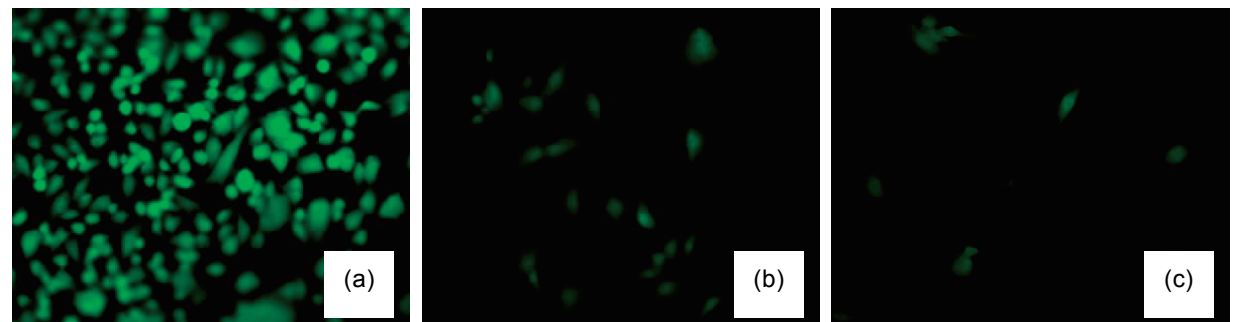

图 4 PMMA 表面修饰前后第 5 天时细胞黏附形态

（a) 未处理; (b) 氨基化后; (c) 水蛭素修饰后 
且表面粗糙度的增大, 也促使蛋白质的吸附较多. 而 接枝水蛭素后材料表面 Fn 在水蛭素接枝后的 PMMA 表面吸附层比较疏松、不稳定, 容易被解吸附, 为可 逆吸附过程; 表面 Fn 质量吸附大大减少.
综上所述，经过氨基化处理后的材料表面修饰 水蛭素药物可以提高亲水性, 并抑制纤连蛋白和细 胞的黏附及增殖, 为临床预防 IOL 二次浑浊提供了 实验依据。

\section{参考文献}

1 Wei Y, Chen Y, Liu P, et al. Surface modification of hydrophobic PMMA intraocular lens by the immobilization of hydroxyethyl methacrylate for improving application in ophthalmology. Plasma Chem Plasma P, 2011, 31: 811-825

2 Zhang L, Wu D, Chen Y, et al. Surface modification of polymethyl methacrylate intraocular lenses by plasma for improvement of antithrombogenicity and transmittance. Appl Surf Sci, 2009, 255: 6840-6845

3 欧瑜, 朱丹丹, 耿培, 等. 重组水蛭素III对半乳糖损伤的人晶状体上皮细胞热应激蛋白表达的影响. 药物生物技术, 2011，18: $115-118$

4 Nakabayashi N. Biomedical application of polymers. Oyo Buturi, 1982, 51: 1189-1193

5 Zheng Z W, Ren L, Feng W J, et al. Surface characterization of polyethylene terephthalate films treated by ammonia low-temperature plasma. Appl Surf Sci, 2012, 258: 7207-7212

6 王瑶, 刘振梅, 徐志康, 等. 聚丙烯酸酯人工晶状体的表面改性研究_常压介质阻挡放电等离子体处理. 中国科学 $\mathrm{E}$ 辑: 技术科学, 2009, 39: 135-143

7 D'sa R A, Burke G A, Meenan B J. Lens epithelial cell response to atmospheric pressure plasma modified poly(methyl methacrylate) surfaces. J Mater Sci-Mater M, 2010, 21: 1703-1712

8 曲超, 姚克, 寇瑞强, 等. 烯丙基葡糖苷对聚甲基丙烯酸甲酯人工晶状体的表面修饰. 生物医学工程学杂志, 2004; 21: 115-117

9 Yao K, Huang X D, Huang X J, et al. Improvement of the surface biocompatibility of silicone intraocular lens by the plasma-induced tethering of phospholipid moieties. J Biomed Mater Res Part A, 2006, 78: 684-692

10 Huang X D, Yao K, Zhang H, et al. Surface modification of silicone intraocular lens by 2-methacryloyloxyethyl phosphoryl-choline binding to reduce Staphylococcus epidermidis adherence. Clin Exp Ophthalmol, 2007, 35: 462-467

11 Mccoy C P, Craig R A, Mcglinchey S M, et al. Surface localisation of photosensitisers on intraocular lens biomaterials for prevention of infectious endophthalmitis and retinal protection. Biomaterials, 2012, 33: 7952-7958

12 Ashraf F T, Atef M, Ahmed A R, et al. Elucidation of antibiotic effectiveness against Staphylococcus epidermidis during intraocular lens implantation. Int J Antimicrob Ag, 2001, 18: 55-59

13 Philipson B, Fagerholm P, Calel B, et al. Heparin surface modified intraocular lenes-a one-year follow-up of a safety study. Acta Opthalmol, 1990, 68: 601-603

14 Kang S, Kim M J, Park S H, et al. Comparison of clinical results between heparin surface modified hydrophilic and hydrophobic acrylic IOLs. Eur J Ophthalmol, 2008, 18: 377-383

15 Kang S, Choi J A, Joo C K. Comparison of posterior capsular opacification in heparin-surface-modified hydrophilic acrylic and hydrophobic acrylic intraocular lenses. Jpn J ophthalmol, 2009, 53: 204-208

16 Demina T, Zaytseva-Zotova D, Yablokov M, et al. DC discharge plasma modification of chitosan/gelatin/PLLA films: Surface properties chemical structure and cell affinity. Surf Coat Tech, 2012, 207: 508-516

17 Marcela M M, Bilek D V B, Alexey K. Free radical functionalization of surfaces to prevent adverse responses to biomedical devices. Proc Natl Acad Sci, 2011, 108: 14405-14410

18 Mittra R A, Dev S, Nasir M A, et al. Recombinant hirudin prevents postoperative fibrin formation after experimental cataract surgery. Ophthalmol, 1997, 104: 558-561

19 Nasir M A, Toth C A, Mittra R A. Recombinant hirudin for prevention of experimental postoperative intraocular fibrin. Am J Ophthalmol, 1996, 121: 554-560 
20 Boeve T J, Reed G L, De Oliveira N C, et al. Comparison of argatroban and hirudin for the reperfusion of thrombotic arterial occlusion by tissue plasminogen activator. J Thromb Thrombolys, 1998, 6: 103-108

21 Lubenow N, Greinacher A. Hirudin in heparin-induced thrombocytopenia. Semin Thromb Hemost, 2002, 28: 431-438

\title{
Anticoagulant r-hirudin coating on PMMA intraocular len surface and its nonfouling properties
}

\author{
ZHENG ZhiWen ${ }^{1,2}$, JIAO Yan ${ }^{1,2}$, REN Li $^{1,2} \&$ WANG YingJun ${ }^{1,2}$ \\ ${ }^{1}$ School of Materials Science and Engineering, South China University of Technology, Guangzhou 510641, China; \\ ${ }^{2}$ National Engineering Research Center for Tissue Restoration and Reconstruction, Guangzhou 510006, China
}

Poly(methyl methacrylate) (PMMA) intraocular len (IOL) surface was treated with ammonia plasma first, followed by coating an anticoagulant drug of r-hirudin. The physical and chemical properties of these surfaces were characterized by the static contact angle, X-ray photoelectron spectroscopy and quartz crystal microbalance. Hydrophilicity of PMMA with r-hirudin coating was improved obviously. Biocompatibility of the samples was tested with HLEpiC. The r-hirudin coated IOLs inhibited the adhesion of fibronectin and reduced the proliferation of HLEpiC evidently, compared to untreated and ammonia plasma treated IOLs. This coating was expected to be used in clinic to prevent the formation of proliferation films and the occurrence of turbidity of IOLs.

polymethacrylate, intraocular lens, ammonia plasma, r-hirudin, protein nonfouling

doi: 10.1360/092013-1149 